\title{
Manipulating miRNA \\ responsiveness to decrease \\ viral pathogenicity
}

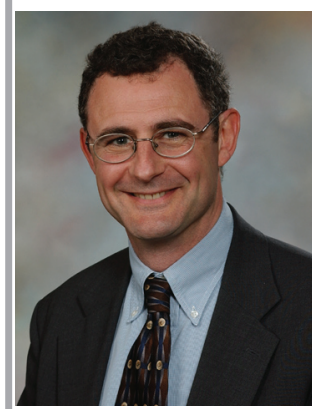

\author{
Stephen J Russell speaks to Shreeya Nanda, \\ Commissioning Editor
}

\section{Stephen J Russell, MD, PhD}

Department of Molecular Medicine \& Division of Hematology, Mayo Clinic,

200 First Street SW, Rochester, MN 55905, USA = Tel.. + 75072848384

- Fax:+7 5072848388 - sjr@mayo.edu

Stephen J Russell is currently Professor of Medicine and Richard $\bigcirc$ Jacobson Professor of Molecular Medicine at the Mayo Foundation in Rochester, Minnesota. He obtained his PhD from the University of London, UK and received specialist accreditation in Hematology from the Royal College of Pathologists. In 1998, he took the position of Director of the Molecular Medicine Program at the Mayo Clinic in Rochester, Minnesota. His research interests focus on developing injectable gene therapy vectors for the treatment of disseminated malignancies (particularly multiple myeloma) and demonstrating their efficacy in clinical trials. Laboratory research themes include the engineering of viral membrane glycoproteins, targeting cell-cell fusion and virus entry, pharmacokinetic and pharmacodynamic studies of virus-based therapies, noninvasive monitoring and imaging of viral gene expression in vivo, and radiovirotherapy. His major research thrust is the development of targetable, injectable oncolytic measles viruses for the treatment of multiple myeloma, ovarian cancer, pancreatic cancer and glioma. Vectors derived from retrovirus, lentivirus, adenovirus, alphavirus and nonviral vectors are also studied in his laboratory.

\section{Can you tell us something about your research background?}

I trained in medicine at Edinburgh University in Scotland and qualified with an MD, an MBChB degree actually, in 1982. I then got interested in hematology but I wanted to do research to try and develop something better for the treatment of patients with leukemia, whose care I was very much involved in. Thus, in 1987, I went to the Chester Beatty laboratories in London, which are the research laboratories for the Marsden hospital and I undertook my $\mathrm{PhD}$ training there. It was in the laboratory of Mary Collins and the Chester Beatty laboratories were very heavily involved in HIV research at the time-CD4 had been identified as the HIV receptor there. My project at that time was to develop retroviral vectors that could be used for cancer therapy. I got my $\mathrm{PhD}$ in 1990 and subsequently moved to Cambridge, where I worked in the Laboratory for Molecular Biology, which is a very highly rated research institution in the UK. On completing my research training and my clinical training, I started to establish my own laboratory. Then
10 years ago, I was recruited to Mayo clinic (MN, USA) to lead a gene therapy effort and it was a very big, well-funded opportunity and I have been here ever since, essentially working on the development of engineered viruses for the treatment of cancer.

\section{In recent times, viruses - long considered exclusively as disease-causing agents - are being used as cancer therapeutics; what is the basis of using viruses in this way?}

Viruses destroy tissue and very often they do so with a high degree of specificity, for example, HIV destroying $\mathrm{CD}^{+}$lymphocytes causing immune deficiency, the hepatitis viruses destroying hepatocytes and so on. The concept has been around for a long time that it might be possible to generate viruses that selectively destroy tumor tissue, using those natural mechanisms of tissue destruction that viruses normally engage against the tissues that they invade. Really the whole idea of using viruses for cancer therapy goes back to the beginning of the last century, and right through the last century there were many experiments in which newly isolated viruses 
were administered to patients with cancer in an attempt to bring about remission of their disease, and in some cases, with certain viruses, the results were quite promising. But I think the whole field got eclipsed when chemotherapy was developed for cancer. In the 1950s and 1960s it looked as if small molecules were going to have a huge impact on cancer care, but we know to our detriment now that that has not been curative in many cases. Thus, with the advances in virology that occurred throughout the last century, and particularly towards the end of the last century when it became possible to rescue viruses from cloned DNA, engineer them and purify them, they started to look appealing again as drugs. There was a revival in the field of developing viruses for cancer therapy again in the 1990s and then throughout the first years of this century as well.

\section{What are the risks involved?}

We all know viruses are inherently risky things since they do cause disease and if a population is not immune, if there is no herd immunity to a virus, then it can spread rapidly through a population, which clearly is the worst case scenario of something that we would wish to avoid when developing viruses for use as therapy. We know that it can be done since there are many live viral vaccines that are in routine use at the moment such as the MMR vaccine, which we give to all our children. But we have to be careful about which viruses we use and how we engineer them in order to avoid any risk of emergence of a virus that is pathogenic and capable of spreading from person to person. There is a great deal of detail in terms of the way in which the field is regulated, what constitutes permissible engineering and what does not, and so we do have to be careful. The major risk is the risk of generating a pathogenic virus that can harm the treated individual and which could theoretically spread to untreated individuals.

\section{Could you briefly explain your new work about using miRNAs to alter viral pathogenicity?}

miRNAs already exist in all cells in the body; many of those miRNAs are tissue specific, therefore they appear only in certain tissues and their abundance varies from tissue to tissue. They are very small segments of RNA - approximately 20-22 bases long - and they have the activity of being able to either interfere with the expression of mRNA) to which they hybridize, or if they hybridize perfectly they can mediate the destruction of those
mRNAs. They are a kind of recently discovered mechanism of regulation of gene expression and what they do is that they modulate the longevity of all the mRNAs that are produced in the cell, depending on their recognition capability. They are present in cells and they are, therefore, potentially available for us to use, and the way in which we use them is to engineer into a virus a target sequence that is recognized by a cellular miRNA such that now when the virus enters the cell expressing that miRNA target sequence its genome is destroyed. This is the whole basis of how we have been able to control virus tropism.

The scenario that we used to demonstrate this utilized a picornavirus called Coxsackie virus A21, a positive-strand RNA virus related to the polio virus, which we found in animal models was able to cause very rapid regression of myeloma tumors grown in mice but then went on to kill the mice by causing myositis, inflammation of the muscles. The virus had two tropisms, one was for tumor and the other was for normal muscle. What we wanted to do was eliminate the tropism for muscle while retaining the ability to propagate and destroy tumor and what we did was introduce miRNA target sequences into the viral genome that were recognized by miRNAs expressed only in muscle. When we studied that virus in vivo we found that, in contrast to the virus from which it was derived, it could destroy the tumors very rapidly but then did not go on to kill the animals and did not cause the myositis. That is essentially what this recent paper was about [1].

\section{What would be the implications for viral therapeutics \& vaccine development?}

The implication for viral therapeutics is that any virus that has the ability to destroy a given tumor type but is associated with an unacceptable toxicity to a normal organ can be engineered by this mechanism to lose its toxicity against the normal tissue while retaining its toxicity to tumors. It may be a general way of developing more highly specific oncolytic, cancer-destroying viruses.

With regard to the vaccine field, the process of developing a vaccine involves either culturing or engineering a virus in some way so it loses its pathogenicity while retaining its ability to stimulate the immune response, and if the pathogenicity is due to damage to a particular tissue then we can use this miRNA engineering approach to eliminate that ability to damage the tissue of interest while retaining its ability to stimulate an immune response. Although this has not been done yet, in theory it can be used to turn a pathogen into a vaccine or even to improve an existing 
vaccine. The polio vaccine, for example, can revert to wild-type and cause polio, and it might then be possible to engineer target sequences that will prevent the polio revertant from infecting the brain, by using neuron-specific miRNA target sequences. Therefore, I think it does have implications for vaccinology as well.

\section{Is this technique applicable to different types of viruses?}

I think it is applicable to other viruses. Viruses enter cells and express their genes in the form of mRNA and thus all of those mRNAs can be engineered to make them susceptible to a given miRNA within a cell. Also for RNA viruses, as in the case of the virus that we studied here, it is possible to insert a target sequence into the viral genome such that the entire viral genome gets degraded. There is no reason why it should not be applicable right across the spectrum of all viruses.

\section{Can you tell us about the other projects that you are involved in?}

The whole thrust of my laboratory, and of quite a few laboratories within the department here, is to develop viruses that can be used for the treatment of cancer. We work with a number of other viruses, like measles virus, vesicular stomatitis virus, adenovirus and a couple of others. One of the overarching problems that we face when attempting to treat cancer patients with these viruses, either engineered or not, is the host immune response to the virus. A preexisting immune response may prevent efficacy altogether or if it is a response to the first dose of virus it may prevent efficacy from subsequent doses. The immune response against the virus in this particular clinical scenario is a big problem and we are working on strategies whereby we can suppress immunity to enhance the activity of the viruses or whereby we can coat the viruses with something to allow them to evade the immune response or even load the viruses into cells that are capable of trafficking to various places in the body and use the cells as delivery vehicles to carry the virus to its target site whilst avoiding neutralization in the bloodstream. Our whole focus is on how we can use viruses for the treatment of cancer.

\section{What are your long-term aims \& where do you think your efforts will be focused over the next 5 to 10 years?}

The long term goal is quite simple for me; it is not a scientific goal, rather it is an applications goal. I want to cure cancer with viruses. As far as I am concerned it is possible since in immunecompromised mice we can give viruses that lead to complete tumor destruction without harming the mouse; but in humans there are many other issues to consider such as the immune system. We are just going to keep pushing in that direction and what I hope to see within the next 10 years is a situation where we really can make a significant impact on the outlook for cancer patients by using a virus.

\section{Financial \& competing interests disclosure}

SJ Russell is a co-inventor on patents (owned by Mayo Clinic) covering the use of measles virus as an oncolytic agent. The author has no other relevant affiliations or financial involvement with any organization or entity with a financial interest in or financial conflict with the subject matter or materials discussed in the manuscript apart from those disclosed.

No writing assistance was utilized in the production of this manuscript.

\section{Bibliography}

1. Kelly EJ, Hadac EM, Greiner S, Russell SJ: Engineering microRNA responsiveness to decrease virus pathogenicity. Nat. Med. 14(11), 1278-1283 (2008).

\author{
Affiliation \\ - Stephen J Russell, MD, PhD \\ Chair, Department of Molecular Medicine \\ and Consultant, Division of Hematology, \\ Mayo Clinic; Professor of Medicine, \\ College of Medicine, 200 First Street SW, \\ Rochester, MN 55905, USA \\ Tel.: +1 507284 8384; \\ Fax: +1 5072848388 ; \\ sjr@mayo.edu
}

